\title{
COMMISSIONING OF NEW SYNCHROTRON RADIATION FACILITIES
}

\author{
Z. T. Zhao \\ Shanghai Institute of Applied Physics, Shanghai 201800, P. R. China
}

\section{Abstract}

Several new synchrotron radiation facilities have been commissioned over the past two years, and almost every commissioning has been an impressive success, reaching high performance very quickly. In this paper, an overview of the new synchrotron radiation facilities that are coming into operation, including Diamond, SOLEIL, the Australian Synchrotron and Indus-2 is presented.

\section{INTRODUCTION}

Over the past three decades, design and construction of storage ring-based synchrotron radiation facilities have been continuously carried out all over the world, starting from second generation light sources in the mid-1970s and focusing on third generation machines after the mid1980s. Since the mid-1990s, the construction of intermediate energy third generation light sources has been the focus of efforts worldwide [1]. Meanwhile, quite a number of compact synchrotron facilities has been developed for various purposes [2]. Currently there are about 50 synchrotron radiation facilities in operation in the world, and about 20 of them are third generation light sources. Furthermore another 10 intermediate energy light sources are in various development stages, from commissioning and construction to advanced design and proposal, which will all be operational before the mid2010s.

To meet users' growing demands for brilliance and time structure of the synchrotron radiation pulse, facilities have been continuously upgraded since the beginning of operation. These upgrades have improved facility performance in many ways, which include (a) increasing brilliance and flux by reducing beam emittance [3] and raising beam current; (b) improving beam orbit stability to micron or sub-micron level [4] and increasing beam current stability by extending beam lifetimes and/or topup injection[5]; (c) producing various polarizations of VUV and X-ray radiation [6]; (e) pushing up the photon energy by using superbends or high harmonics of undulators [6]; (f) generating picosecond or even subpicosecond X-ray pulses [7] or coherent synchrotron radiation [8]; and (g) increasing the number of ID beamlines via canted undulator scheme.

Along with the accelerator R\&D progress, many new technologies have been tested and applied in the existing and new facilities, such as novel insertion devices (IDs), including electromagnetic undulators, pure permanent and hybrid undulators, small gap in-vacuum undulators to superconducting IDs; beam and orbit feedback systems [9]; superconducting cavities and superconducting magnets [10]; digital BPM processing systems [11], and digital power supply and low level RF controllers [12]. Performance goals for new synchrotron radiation facilities are defined based on the experience with construction and upgrade at existing facilities.

\section{NEW SYNCHROTRON FACILITIES}

Storage ring-based light source facilities are the main workhorses serving the SR user community. Even though FEL and ERL light source are on the horizon, the new development of storage ring-based light sources is still very strong. Table 1 lists the third generation sources in operation, under construction and being proposed.

\section{Intermediate energy light sources}

From Table 1, one can see that most new synchrotron radiation facilities are third generation, low emittance light sources in the intermediate energy range $(2.5-3.5$ $\mathrm{GeV})[13,14]$, which can produce high brilliance hard Xrays up to $20 \mathrm{keV}$ by employing the higher harmonics of small gap, in-vacuum undulators.

Since the beginning of $21^{\text {st }}$ century, four intermediate energy synchrotron radiation facilities have been successively put into operation: the SLS in 2001, ANKA in 2002, CLS in 2003, and SPEAR3 in 2004. Another four, Diamond [15], SOLEIL [16], ASP [17] and Indus-2 [18] are becoming operational this year. In addition, a partially dedicated intermediate energy synchrotron radiation facility, BSRF/BEPC-II, was put into operation in 2006. Three more facilities will be in operation in four years: the SSRF [19] in 2009, ALBA [20] in 2010 and probably SESAME [21] in 2011. A new group of lower emittance intermediate energy synchrotron facilities, including NSLS-II [22], TPS [23] and MAX-IV [24], are in solid project or proposal stage, and they are expected to be operational before 2015. There are a few more new intermediate energy facility plans under consideration in Armenia, Poland and South Africa [25].

\section{Low and high energy light sources}

General-purpose low energy synchrotron light sources are developed in various ways. Two low energy light sources, the 1.5-GeV SAGA-LS [26] and the 700-MeV MAX-III [27], became operational in 2005 and this year respectively. Another $600-\mathrm{MeV}$ light source, MLS [28] for metrology applications, is in commissioning in Berlin.

A number of new low energy light sources are in the design stage. MAX-IV includes a $1.5-\mathrm{GeV}$ light source with an emittance of lower than $0.5 \mathrm{~nm}$-rad, and three low energy light sources are being proposed by INP in Republic of Kazakhstan [29], Nagoya University [30] and Tohoku University [31] in Japan. 
Table 1: Main parameters of the third generation light sources

\begin{tabular}{|c|c|c|c|c|c|c|}
\hline Light Source & $\begin{array}{l}\text { Energy } \\
(\mathrm{GeV})\end{array}$ & $\begin{array}{c}\text { Circumference } \\
\text { (m) }\end{array}$ & $\begin{array}{l}\text { Emittance } \\
\text { (nm.rad) }\end{array}$ & $\begin{array}{c}\text { Current } \\
(\mathrm{mA})\end{array}$ & Straight Section & Status \\
\hline ESRF & 6.0 & 844.4 & 3.7 & 200 & $32 \times 6.3 \mathrm{~m}$ & Operational(1993) \\
\hline APS & 7.0 & 1104 & 3.0 & 100 & $40 \times 6.7 \mathrm{~m}$ & Operational(1996) \\
\hline SPring-8 & 8.0 & 1436 & 2.8 & 100 & $44 \times 6.6 \mathrm{~m}, 4 \times 30 \mathrm{~m}$ & Operational(1997) \\
\hline ALS & 1.9 & 196.8 & 6.3 & 400 & $12 \times 6.7 \mathrm{~m}$ & Operational(1993) \\
\hline TLS & 1.5 & 120 & 25 & 240 & $6 \times 6 \mathrm{~m}$ & Operational(1993) \\
\hline ELETTRA & $2.0 / 2.4$ & 259 & 7 & 300 & $12 \times 6.1 \mathrm{~m}$ & Operational(1994) \\
\hline PLS & 2.5 & 280.56 & 10.3 & 200 & $12 \times 6.8 \mathrm{~m}$ & Operational(1995) \\
\hline LNLS & 1.37 & 93.2 & 70 & 250 & $6 \times 3 \mathrm{~m}$ & Operational(1997) \\
\hline MAX-II & 1.5 & 90 & 9.0 & 200 & $10 \times 3.2 \mathrm{~m}$ & Operational(1997) \\
\hline BESSY-II & 1.7 & 240 & 6.1 & 200 & $8 \times 5.7 \mathrm{~m}, 8 \times 4.9 \mathrm{~m}$ & Operational(1999) \\
\hline Siberia-II & 2.5 & 124 & 65 & 200 & $12 \times 3 \mathrm{~m}$ & Operational(1999) \\
\hline NewSUBARU & 1.5 & 118.7 & 67 & 500 & $2 \times 14 \mathrm{~m}, 4 \times 4 \mathrm{~m}$ & Operational(2000) \\
\hline SLS & $2.4-2.7$ & 288 & 5 & 400 & $3 \times 11.7 \mathrm{~m}, 3 \times 7 \mathrm{~m}, 6 \times 4 \mathrm{~m}$ & Operational(2001) \\
\hline ANKA & 2.5 & 110.4 & 50 & 200 & $4 \times 5.6 \mathrm{~m}, 4 \times 2.2 \mathrm{~m}$ & Operational(2002) \\
\hline CLS & 2.9 & 170.88 & 18.1 & 500 & $12 \times 5.2 \mathrm{~m}$ & Operational(2003) \\
\hline SPEAR-3 & 3.0 & 234 & 12 & 500 & $2 \times 7.6 \mathrm{~m}, 4 \times 4.8 \mathrm{~m}, 12 \times 3.1 \mathrm{~m}$ & Operational(2004) \\
\hline SAGA-LS & 1.4 & 75.6 & 7.5 & 300 & $8 \times 2.93 \mathrm{~m}$ & Operational(2005) \\
\hline ASP & 3.0 & 216 & $7-16$ & 200 & $14 \times 5.4 \mathrm{~m}$ & Commis \& Oper \\
\hline Indus-II & 2.5 & 172.5 & 58 & 300 & $8 \times 4.5 \mathrm{~m}$ & Commis \& Oper \\
\hline Diamond & 3.0 & 561.6 & 2.7 & 300 & $6 \times 8 \mathrm{~m}, 18 \times 5 \mathrm{~m}$ & Commis \& Oper \\
\hline Soleil & 2.75 & 354.1 & 3.74 & 500 & $4 \times 12 \mathrm{~m}, 12 \times 7 \mathrm{~m}, 8 \times 3.8 \mathrm{~m}$ & Commis \& Oper \\
\hline PETRA-III & 6.0 & 2304 & 1.0 & 100 & $1 \times 20 \mathrm{~m}, 8 \times 5 \mathrm{~m}$ & Construction \\
\hline SSRF & 3.0 & 432 & 3.9 & 300 & $4 \times 12 \mathrm{~m}, 16 \times 6.5 \mathrm{~m}$ & Construction \\
\hline ALBA & 3.0 & 268.8 & 4.5 & 400 & $4 \times 8 \mathrm{~m}, 12 \times 4.2 \mathrm{~m}, 8 \times 2.6 \mathrm{~m}$ & Construction \\
\hline SESAME & 2.5 & 133.12 & 26 & 400 & $8 \times 4.44 \mathrm{~m}, 8 \times 2.38 \mathrm{~m}$ & Construction \\
\hline CANDLE & 3.0 & 216 & 8.4 & 350 & $16 \times 4.8 \mathrm{~m}$ & Planned \\
\hline MAX IV & $1.5 / 3.0$ & 287.2 & $0.34 / 0.8$ & 500 & $12 \times 4.6 \mathrm{~m}$ & Planned \\
\hline NSLS-II & 3.0 & 780 & 2.1 & 500 & $15 \times 8 \mathrm{~m}, 15 \times 5 \mathrm{~m}$ & Planned \\
\hline TPS & 3.0 & 518.4 & 1.7 & 400 & $6 \times 11.7 \mathrm{~m}, 18 \times 7 \mathrm{~m}$ & Planned \\
\hline TSRF & 1.8 & 244.8 & 4.9 & 400 & $2 \times 15,14 \times 5 \mathrm{~m}$ & Planned \\
\hline Kazakhstan LS & 2.0 & 135 & 10 & 500 & $6 \times 7 \mathrm{~m}$ & Planned \\
\hline
\end{tabular}

New high energy synchrotron light sources may be a practical option if one considers the future conversion of the existing high energy physics machines. As a model, PETRA-III [32], a 6-GeV, 1 nm-rad light source converted from a former high energy physics machine, is under construction at DESY. It is scheduled to be operational in 2009.

\section{Ultimate storage ring light sources}

Beyond the light source construction projects and plans mentioned above, many attempts have been made to push up the performance limits of the storage ring based synchrotron radiation facilities. Studies were conducted to investigate the ultimate performance limits and technical challenges for constructing next generation light source storage rings at the ESRF [33], APS [34] and SPring-8 [35]. Having an energy of 6 or $7 \mathrm{GeV}$, these storage rings are designed to have a natural emittance in the range of 0.1-0.3 nm-rad and a beam current of $500 \mathrm{~mA}$, which aims at increasing the brilliance of presently operating facilities by two orders of magnitude.

Although storage rings with very low emittance suffer from challenges of high heat loads, small dynamic aperture and short beam lifetime, the designs indicate that it is possible to construct and operate such a machine by using top-up injection, orbit feedback and damping wigglers or even longitudinally varying dipole field to reduce emittance.

\section{COMMISSIONING OF NEW FACILITIES}

\section{Diamond [15,36]}

Diamond is the UK's new third generation light source located at Oxfordshire. Its construction started in 2002, accelerator installation started in Winter 2004 and the facility was completed in Apr. 2006.

The commissioning of the $100-\mathrm{MeV}$ Diamond linac was completed with all parameters within specifications in mid-October, 2005. Then the booster commissioning started on Dec. 19, 2005, with first injection attempts at a fixed booster energy of $100-\mathrm{MeV}$. The first turn of the beam was achieved on the night of Dec. 21. Because the restriction of cooling water at that time, the maxiumum beam energy of $700-\mathrm{MeV}$ was allowed to be obtained for the booster. The first acceleration of $700 \mathrm{MeV}$ was achieved on Mar. 10, 2006 and the first extraction of the $700-\mathrm{MeV}$ beam was achieved on Apr. 4. 3-GeV beam acceleration and extraction were achieved on June 9. 
The Diamond storage ring commissioning started at $700-\mathrm{MeV}$ as well. The first injection was made on May 4, 2006 and the first turn was achieved on May 5. A stored beam of $0.5 \mathrm{~mA}$ was obtained on May 22 and 2-mA beam was stacked on May 30 .

The 3-GeV Diamond storage ring commissioning started on Sept. 4, and 2-mA stored beam was achieved on Sept. 6. One month later, a 90-mA stored beam was obtained and beam line commissioning started. The first light was let into a beam line hutch on Oct. 12. In the following months, 100-mA stored beam was achieved on Nov. 11 and $150 \mathrm{~mA}$ on Jan. 12, 2007. Now there are a total of eight commissioned IDs (6 in-vacuum undulators, one APPLE-II and one SC wiggler).

\section{SOLEIL[16,37]}

SOLEIL is the new French synchrotron radiation facility located at St. Aubin near Paris. Its construction started in January 2002 and the machine installation was completed in May 2006.

The $100-\mathrm{MeV}$ SOLEIL electron linac commissioning was completed with performance within specifications and its acceptance was announced on Nov. 15, 2005. The first injection of the $110-\mathrm{MeV}$ beam to booster occurred on July 15,2005 and the first acceleration of the beam to $2.75 \mathrm{GeV}$ was made on Oct.13, 2005. Beam extraction and transport from booster to storage ring occurred on May 8, 2006.

The first beam injection to the storage ring was made on May 14, 2006, the first stored beam was achieved on June 4 and 100-mA beam was obtained on July 4. 300 $\mathrm{mA}$ was stored in a $3 / 4$ full bunch filling pattern on September 25, 2006 after eight effective weeks of commissioning. From July 13 to Dec. 13, first photon beams were delivered to five beam lines ( 2 ID and 3 bend lines). By the end of June, 2007, there were ten IDs installed and commissioned, including three in-vacuum undulators, three APPLE-II type undulators and four electromagnetic undulators.

\section{Australian Synchrotron [17,38]}

The Australian synchrotron is a third generation light source located adjacent to Monash University in Clayton, Victoria. Construction started in 2003 and the storage ring installation finished in May 2006.

The commissioning of the turn-key injector system, including a $100-\mathrm{MeV}$ electron linac and a $3-\mathrm{GeV}$ booster synchrotron, started in Oct. 2005, and the first electron beam accelerated through the linac was observed in the transfer line in mid-December 2005. The first beam was injected into the booster in Feb. 2006 and the first beam was accelerated to $3 \mathrm{GeV}$ in the beginning of April.

The storage ring commissioning started with the commissioning of the booster to storage ring (BTS) transfer line, and the first beam in the BTS section was observed on June 1, 2006. Then the first turn in the storage ring was achieved on June 8, and the first beam was stored and stacked to $1 \mathrm{~mA}$ on July 14 . The acceptance beam current of $100 \mathrm{~mA}$ for the project was obtained in mid-November and the target beam current of $200 \mathrm{~mA}$ was achieved on Dec. 15, 2006.

\section{Indus-2[18]}

Indus- 2 is an Indian third generation light source set up at RRCAT, Indore. Its construction started in 1998, and its subsystem installation has been carried out from 2003-04 onwards.

The commissioning of the Indus-2 transfer line (TL-3) from the existing booster to the storage ring was carried out in May 2005. The first electron beam was injected into the storage ring in Aug. 2005, a four-turn beam circulation in the storage ring was achieved on Aug. 27, and the first light out of Indus-2 was recorded on Dec. 2, 2005. Beam was accumulated in January 2006 and the first beam of a few milliamperes was stored in Feb. 2006. The beam was successfully ramped from $450 \mathrm{MeV}$ to 2 $\mathrm{GeV}$ and $2.4 \mathrm{GeV}$ in May-June 2006. Since then two beam lines, X-ray diffraction (XRD) and extended X-ray absorption fine structure, have been installed and commissioned, and the first record of X-ray diffraction with SR was achieved on Sept. 28, 2006.

\section{SSRF [19]}

SSRF is a third generation light source located in Pudong Zhangjiang High-Tech Park, Shanghai. The SSRF construction was launched in Dec. 2004. Less than two years later, installation of the linac started and the booster and storage ring are presently being installed.

The commissioning of the $150-\mathrm{MeV}$ SSRF linac started on May 15, 2007, and the first beam was seen with the linac diagnostics on the same day. The required energy, energy spread, current and emittance have been obtained, and now the commissioning to meet full linac specifications is being carried out and is expected to be completed this August. The booster commissioning is scheduled to start in Oct., and the ring commissioning is planned to begin in Apr. 2008. However, an early start of the commissioning is expected.

\section{$M A X$-III and BEPC-II}

These two machines represent the compact and the partially dedicated synchrotron radiation facilities. MAX-III is a compact light source optimised for the UV and IR spectral region with a circumference of $36 \mathrm{~m}$ and energy of $700 \mathrm{MeV}$. It has very unique solid iron cell blocks containing all magnets [27]. The MAX-III storage ring commissioning started in 2006 and, with the help of a $5^{\text {th }}$ harmonic Landau cavity, a circulating beam of $350 \mathrm{~mA}$ was achieved.

The new 2.5-GeV BSRF storage ring in Beijing is comprised of two outer halves of the BEPC-II electron ring and positron rings and is a partially dedicated SR source[39]. The BSRF ring commissioning started with an injection energy of $1.89 \mathrm{GeV}$ from the IHEP linac on Nov. 13, 2006, and a one-turn beam signal was observed on the first day. The first stored beam was obtained on Nov. 18, and $\sim 100 \mathrm{~mA}$ at a ramped energy of $2.5 \mathrm{GeV}$ was achieved on Dec. 252006. 


\section{COMMISSIONING APPROACHES}

Injector systems that include an electron linac and booster synchrotron can be constructed either in-house with the storage ring or through turn-key contracts. Injector commissioning is typically fast, involving a joint effort with the vendor in the turn-key case, and, in most cases, the performance is up to or better than its technical specifications. On the other hand, the commissioning of most new storage rings is an integrated effort based on worldwide collaboration. The commissioning of many new facilities benefits from the techniques and application software developed over the last 10 years at the ALS and SSRL [40-43]. Present-day commissioning is typically very efficient, where the beam is stored and accumulated very quickly and machine performance is easily characterized. In the following, a number of storage ring commissioning approaches are summarized.

\section{Injection and accumulation}

Beam injection, storage and accumulation are the first tasks in storage ring commissioning. These programs characterize the actual quality magnet production and alignment and test the performance of beam diagnostics, controls, power supplies, vacuum system, injection system and RF system. The normal procedures start with first-turn and multi-turn beam circulation with RF, sextupoles and correctors off. To confirm the injection energy and correct the first-turn beam orbit, SOLEIL started beam injection with only bending magnets powered on and deliberate on-axis injection, followed by turning on quadrupoles and correctors. In this stage, firstturn and turn-by-turn BPM capabilities are valuable.

\section{Minimizing and stabilizing closed orbit}

Once the beam is stored in the ring, one can use beambased alignment (BBA) to determine each BPM offset with respect to its nearest quadrupole magnetic center, and SVD-based orbit correction to minimize the closed orbit. After BBA and SVD-based orbit correction, Diamond can reduce its residual closed orbit to less than $1 \mu \mathrm{m}$ by using all 168 correctors in each plane. Using only 96 correctors, Diamond can achieve rms orbit errors of $60 \mu \mathrm{m}$ horizontally and $40 \mu \mathrm{m}$ vertically. At SOLEIL, rms residual orbits of $42 \mu \mathrm{m}$ horizontally and $78 \mu \mathrm{m}$ vertically are achieved by using 56 correctors in each plane. The orbit in ASP ring has been corrected to an rms deviation of $\sim 16 \mu \mathrm{m}$.

The measurement of orbit stability is an important commissioning task. One needs to characterize the slow orbit drift correlation with thermal, mechanical and electrical factors, and measure the orbit's power spectral density (PSD) to identify the disturbing sources. Diamond and SOLEIL have commissioned their slow orbit feedbacks for obtaining micron or even sub-micron orbit stability. With orbit feedback in operation, the integrated vertical position stability is $0.5 \mu \mathrm{m} \mathrm{rms}$ for Diamond and less than $2 \mu \mathrm{m}$ rms for SOLEIL. Fast orbit feedback has been commissioned at Diamond, reducing the integrated noise from $4 \mu \mathrm{m}$ to $1 \mu \mathrm{m}$ horizontally and from $1 \mu \mathrm{m}$ to $0.4 \mu \mathrm{m}$ vertically, up to $100 \mathrm{~Hz}$.

\section{Optics and machine characterization}

Measurement and optimization of the storage ring linear optics are the indispensable commissioning tasks. The LOCO program [41, 42] has become a standard tool to characterize, and correct as needed, real normal and skew quadrupole gradient errors, BPM and corrector gains, insertion device gap compensations, betatron coupling, local chromaticities and transverse impedance distributions, based on the fit of measured and model orbit response matrices. In addition, beam-based and radiation-based measurements of beam parameters, such as emittance, energy spread, lifetime, betatron coupling, etc., are necessary to verify the ring model calibration. The linear optics corrections and machine parameter calibrations of Diamond, SOLEIL and ASP have been all done with LOCO.

\section{Beam current, beam lifetime and instabilities}

As the commissioning time increased, the beam lifetime of the light source storage ring is improved by increasing integrated beam current, the beam lifetime of Diamond, SOLEIL and Australian synchrotron have all exceeded 20 hours at $100 \mathrm{~mA}$

For the new light sources, high current is limited mainly by transverse coupled bunch instabilities caused by resistive wall impedance and ions. Single bunch and multi bunch beam instabilities have been examined at Diamond, SOLEIL and the Australian Synchrotron. The multi bunch vertical threshold currents measured at zero chromaticities are $17 \mathrm{~mA}$ for Diamond and $30 \mathrm{~mA}$ for SOLEIL $[15,16]$. Bunch filling patterns having a gap and increased chromaticities can push up the current threshold of this instability. To date the Australian synchrotron has stored the target current of $200-\mathrm{mA}$, SOLEIL has achieved a 300-mA stored current with 312 of 416 buckets filled and Diamond has obtained 170-mA stored current with only one RF cavity in operation and limited RF conditioning time. In addition, Diamond and SOLEIL have commissioned their transverse beam feedback system [36, 37, 44].

\section{Insertion device commissioning}

Insertion devices can introduce closed orbit distortion, tune shift, beta beating and dynamic aperture degradation that can reduce injection efficiency and even beam lifetime. ID compensation, as determined with LOCO, and feed-forward correction during ID scans are indispensable. In particular, EPUs, which provide full polarization control of photon beam, may introduce intrinsic skew quadrupole fields, causing beam size instability, and other strong nonlinearities that need especially careful treatment.

At Diamond the uncorrected closed orbit change as a function of ID gap is of order 20-25 $\mu \mathrm{m}$ for in-vacuum undulators and $50 \mu \mathrm{m}$ for APPLE-II undulators $[15,45]$. With the feed-forward compensation, a residual orbit 
disturbance of 1-2 $\mu \mathrm{m}$ has been achieved. For HU80 and HU256 IDs at SOLEIL, around $1.5 \mu \mathrm{m}$ rms residual orbit motion is observed with feedforward switched on[16, 46].

\section{Top up operation studies}

Top-up operation makes heat loads for accelerator and beam line components constant, which has been proven to be extremely helpful in improving the stability of the photon beam for SR experiments. The top-up operation for most new facilities is considered in their design stage, and once the basic commissioning with IDs is completed, top-up operation is chosen as the next commissioning task. This year Diamond, SOLEIL and ASP have all performed top-up preparations and machine studies and preliminary results have been achieved [36-38].

\section{CONCLUSIONS}

The development of storage ring-based synchrotron radiation facilities is still active and growing. There will be more than 10 new light sources operational before 2015. The commissioning of recent facilities, Diamond, SOLEIL, ASP and Indus-2, has been successful and valuable experience has been gained that will benefit light source facilities now in construction and planning. Commissioning techniques are mature and efficient, and worldwide collaboration and sharing of experience are very effective.

\section{ACKNOWLEDGEMENTS}

I would like to thank M. Eriksson of MAX-lab, S. Kotaiah of RRCAT, R.P. Walker and R. Bartolini of DLS, G. LeBlanc and M.J. Spencer of ASP, J.M. Filhol and A. Nadji of Soleil, for providing me informative materials on the commissioning results of their new synchrotron facilities.

\section{REFERENCES}

[1] A. L. Robinson, X-Ray Data Booklet, 2000.

[2] Ernst Weihreter, compact synchrotron light sources, World Scientific, 1996.

[3] Small Emittance Light Source Workshop, Lund March 10-12, 2004.

[4] M. Boege, Proc. Of EPAC04, Lucern, July 5-9, 2004, Switzerland, p. 211.

[5] H. Tanaka, J. Synchrotron Rad. (2006) 13, 378-391

[6] J. Chavanne and P. Elleaume, Proc. of EPAC06, Edinburgh, June 26-30, 2006, p. 969.

[7] A. A. Zholents, these proceedings.

[8] M. Abo-Bakr et al., Phys. Rev. Lett. 88, 254801 (2002).

[9] D. Teytelman, Proc. of PAC03, Portland, May 12-14, 2003, p. 318.

[10]Applications of Superconducting Technology in Synchrotron Light Sources, Satellite Workshop of SRI2006, Hsinchu, June 5-6, 2006

[11]A. Kosicek, Proc. of PAC05, Knoxville, May 16-20, 2005, p. 4284
[12]M. E. Angoletta, Proc. of EPAC06, Edinburgh, June 26-30, 2006, p. 1847

[13] Shanghai Symposium on Intermediate-energy Light Sources, September 24-26, 2001, Shanghai, China

[14]J. Corbett and T. Rabedeau, SLAC-PUB-9600, November 2002.

[15]R.P. Walker, Proc. of APAC07, Indore, Jan.29 Feb.2, 2007.

[16]L. Nadolski et al., Proc. of APAC07, Indore, Jan.29 - Feb.2, 2007.

[17]G. LeBlanc, Proc. of APAC07, Indore, Jan.29 Feb.2, 2007.

[18]V. C. Sahni, Proc. of APAC07, Indore, Jan.29 Feb.2, 2007.

[19]Z. T. Zhao et al., Proc .of APAC07, Indore, Jan.29 Feb.2, 2007.

[20]D. Einfeld, these proceedings.

[21]G. Vignola et al, Status of SESAME, EPAC06, Edinburgh, June 26-30, 2006, p. 3383.

[22]S. Ozaki et al, theses proceedings

[23]K. S. Liang et al., APAC07, Indore, Jan.29 - Feb.2, 2007.

[24]M. Eriksson et al., these proceedings.

[25]H. Winick, Private communication.

[26]T. Tomimasu et al., Proc. of PAC05, Knoxville, May 16-20, 2005, p. 4021.

[27]M. Eriksson et al., Proc. of EPAC06, Edinburgh, June 26-30, 2006, p. 3416.

[28]J. Feikes et al., these proceesings.

[29]K. Kadyrzhanov et al, Proc. of RuPAC06, p. 407.

[30]Y. Takashima et al, Proc. of SRI 2006, Gaegu, May 28 - June 2, 2006, p.75.

[31]S. Suzuki et al., Nucl. Inst. And Meth. A 467-468, 72 (2001).

[32]K. Balewski, Proc. of EPAC06, Edinburgh, June 2630, 2006, p. 3317.

[33]A. Ropert et al., Proc. of EPAC00, Vienna, June 26 $-30,2000$, p. 83.

[34]M. Borland, Nucl. Inst. And Meth. A 557, 230 (2005).

[35]K. Tsumaki and N. Kumagai, Proc. of EPAC06, Edinburgh, June 26-30, 2006, p. 3362.

[36]R. Bartolini, these proceedings.

[37]A. Nadji, these proceedings.

[38]A. Jackson, these proceedings.

[39]C. Zhang, Proc. of APAC07, Indore, Jan.29 - Feb.2, 2007.

[40]A. Terebilo, Proc. of PAC01, Chicago, June 18-22, 2001, p3203; SLAC-PUB-8732.

[41]J. Safranek, Nucl. Inst. And Meth. A 388, 27 (1997).

[42]J. Safranek et al, MATLAB-based LOCO, EPAC02, Paris, June 3-7, 2002, p. 1184

[43]G. Portmann, J. Corbett and A. Terebilo, Proc. of PAC05, Knoxville, May 16-20, 2005, p. 4009.

[44]R. Nagaoka et al., these proceedings.

[45]B. Singh, these proceedings.

[46]C. Benabderrahmane, these proceedings. 